\begin{tabular}{|c|l|}
\hline Title & A pplication of the multichannel electrode method to monitoring of corrosion of steel in an artificial crevice \\
\hline Author(s) & Naganuma, A tsushi; Fushimi, Koji; A zumi, Kazuhisa; Habazaki, Hiroki; Konno, Hidetaka \\
\hline Citation & $\begin{array}{l}\text { Corrosion Science, 52(4), 1179-1186 } \\
\text { https://doi.org/10.1016j.corsci.2010.01.005 }\end{array}$ \\
\hline Issue Date & 2010-04 \\
\hline Doc URL & http://hdl.handle.net/2115/43009 \\
\hline Type & article (author version) \\
\hline File Information & CS52-4_1179-1186.pdf \\
\hline
\end{tabular}

Instructions for use 


\section{Application of the Multichannel Electrode Method to Monitoring of Corrosion of Steel in an Artificial Crevice}

Atsushi Naganuma, Koji Fusimi, Kazuhisa Azumi, Hiroki Habazaki, Hidetaka Konno Graduate School of Engineering, Hokkaido University

N13W8, Kitaku, 060-8628 Sapporo, Japan

Tel / Fax: +81-11 -706-6749

e-mail: naga@eng.hokudai.ac.jp

Abstract Crevice corrosion of iron was evaluated using the multichannel electrode method in which ten individual working electrodes (WEs) of pure iron were embedded in resin, placed in an artificial crevice in the range from $0.5 \mathrm{~mm}$ to $2.0 \mathrm{~mm}$, and immersed in $0.51 \mathrm{~mol} \mathrm{dm}^{-3} \mathrm{NaCl}$ solution. The WEs were connected to an electronic circuit which allowed galvanic coupling between them and measurement of their individual coupling current or open circuit potential. Time-transient of the spatial distribution of coupling current and open circuit potential showed sequential transition 
of the coupling current on WEs at the middle position of the crevice from cathode to anode. The WE near the opening of the crevice initially showed a large anodic current, then a decreasing the anodic current corresponding to the current transition of other WEs, and finally a large cathodic current coupled with the other anodic WEs in the crevice. The transition of coupling current was explained by the change in $\mathrm{pH}$ and concentration of dissolved oxygen in the crevice. Thickness of the gap of the artificial crevice affected the transition behavior of coupling current distribution. For example, slower current transition with smaller coupling current was found in the case of a narrower gap. Such properties were related to the introduction and consumption of dissolved oxygen in the crevice solution and the circulation of gap solution from / to the outside of the crevice.

Keyword: B. Multichannel electrode method; C. crevice corrosion; C. coupling current; C. dissolved oxygen

\section{Introduction}


Nonuniform corrosion is widely observed in corroding materials in a practical

situation. Evaluation of such nonuniform corrosion is very important because the

lifetime of materials can be determined by the most corrodible part of nonuniform corrosion. Crevice corrosion is a common and insidious form of localized corrosion that occurs in fissures or occluded regions under, for example, washers and joints [1]. In the laboratory, crevice corrosion has been traditionally studied using methods such as weight-loss measurement, visual and microscopic inspection of corroded specimens, freezing methods using a micro-electrode [2] and simulation of corrosion in the occluded space [3]. Mathematical modeling has also been extensively used for analysis of crevice corrosion [4-8]. Recently, Nagarajan et al. measured crevice corrosion resistance of superausetenitic stainless steel in natural sea water using dynamic electrochemical impedance spectroscopy which revealed changes in passive layer structure [9], Abdulsalam investigated crevice corrosion of iron in acetate solution by measuring current and potential gradient within the crevice in the depth direction, which showed a critical depth value, above which little changes occur on the transition boundary between passive and active region on the crevice wall [10] and Galvele 
reviewed the cretical potential for pitting and crevice corrosion using Tafel's law measurement, which acquired the information of susceptibility to crevice corrosion of metals and alloys in $\mathrm{NaCl}$ solution [11]. Although these methods have provided valuable information concerning the mechanism of crevice corrosion, use of a single specimen restricts evaluation of the corrosion rate spatially distributed in the crevice and thus delineation of the nonuniform crevice corrosion process.

In the general concept proposed for crevice corrosion, heterogeneous electrochemical processes of anodic and cathodic reactions occur on separated areas of the corroding material. Distribution and intensity of anodic and cathodic reactions depend on conditions such as shape and size of the crevice, composition and $\mathrm{pH}$ of the solution in the crevice, and supply of ions and oxygen into the crevice [12, 13]. For quantitative analysis of these parameters, we have developed a multichannel electrode method [14]. In this method, a corrodible metal or alloy is divided into multiple pieces of individual working electrodes, wired and electrically insulated, reconstructed in epoxy resin, and placed in a corrosion environment to measure coupling current between these electrodes and immersion potential of individual electrodes. We applied 
this technique to analysis of the spacial distribution of corrosion rate of welded steel and lifetime prediction of corrosion protection performance of zinc-rich paint on a welded area [14]. This system is similar to the method developed by Tan et al. that is applied to inhomogeneous corrosion under organic coating $[15,16]$, monitoring and estimation of localized corrosion rates [17], mapping of nonuniform corrosion [18] and crevice corrosion [19]. In this study, the multichannel electrode system was applied to corrosion behavior of iron composed of ten electrodes placed in an artificial crevice immersed in sodium chloride solution, and the spacial distributions of anodic and cathodic contribution and its change with immersion time were measured.

\section{Experimental}

Ten specimens were prepared from an iron rod (99.5\% purity, $10 \mathrm{~mm}$ in diameter, $20 \mathrm{~mm}$ in length) for use as working electrodes (WEs). They were wired, coated with electrodeposition paint to prevent crevice corrosion of the side face, and embedded together in epoxy resin with the arrangement shown in Fig. 1. Indication of any crevice corrosion between iron specimen and epoxy resin was not observed 
during experiments. The surface was mechanically abraded together with SiC paper of 1000 grid. Before the immersion test, WEs were passivated at $0.8 \mathrm{~V}_{\mathrm{Ag} / \mathrm{AgCl}}$ in $\mathrm{pH} 8.4$ borate buffer solution $\left(0.15 \mathrm{~mol} \mathrm{dm}^{-3} \mathrm{H}_{3} \mathrm{BO}_{4}+3.74 \times 10^{-2} \mathrm{~mol} \mathrm{dm}^{-3} \mathrm{Na}_{2} \mathrm{~B}_{4} \mathrm{O}_{7} \cdot 10 \mathrm{H}_{2} \mathrm{O}\right)$ to improve reproducibility of experimental data. An artificial crevice with width and length set at $12 \mathrm{~mm}$ and $135 \mathrm{~mm}$ was formed on the surface of WEs by using a transparent acrylic plate and a silicone sheet for spacing. The crevice gap between the WE surface and acrylic plate, $d_{\text {Gap }}$, was adjusted to be $0.5,0.8,1.0,1.5$ and $2.0 \mathrm{~mm}$ using different thickness of silicone sheet. One side of the crevice was exposed to the test solution $\left(0.51 \mathrm{~mol} \mathrm{dm}^{-3} \mathrm{NaCl}\right.$ solution with volume of $\left.6 \mathrm{dm}^{3}\right)$ and the with temperature set at 298 K. Each WE was numbered from 0 at the bottom of the crevice to 9 near the opening. A $\mathrm{Ag} / \mathrm{AgCl} /$ sat. reference electrode (RE) was placed outside the crevice. The WEs were immersed in the test solution for about 240 ks (ca. 3 days), and the corrosion condition of the WEs was recorded using a digital camera at a constant interval. In some experiments, the channel in the acrylic plate was filled with agar containing bromothymol blue (BTB) to show the distribution of $\mathrm{pH}$ in the crevice, respectively. The color of BTB is yellow at $\mathrm{pH}$ lower than 6 and blue at $\mathrm{pH}$ higher than 7.5. 
Fig. 2 shows the electronic circuit of the multichannel electrode system. Each WE was connected to low-resistance photo-MOS relays which route the connection of the WE to ground level (common level) or virtual ground level provided by an operational amplifier (OPA) to measure the electric current between the WE and ground level. In this circuit, electric voltage of two input terminals (+ and -) is kept to be identical. Impedance of two input terminals of OPA is very high and thus the inflow current from WE(n) toward (-) input terminal passes through the resistance to output terminal of OPA. This causes voltage drop at the resistance between (-) input and output terminals which corresponds to the coupling current at WE(n). Current and potential of each WE and RE were measured using a multifunction data acquisition board (National Instruments, model PCI-6229) controlled by a program coded with a LabVIEW software package (National Instruments). Two kinds of measurement were performed during immersion, as shown in Fig. 3. In period A, all WEs were connected to the common (ground) level and thus the electrode potential of all WEs was identical and galvanic coupling between all WEs was established. In this period, one of the WEs was selected in turn and connected to virtual ground of OPA input to measure its coupling 
current. Potential of RE, $E_{\mathrm{RE}}$, located near the opening was also measured in this period.

Immersion potential of the WEs, $E_{\mathrm{A}}$, was thus obtained from the potential difference between the common level and $E_{\mathrm{RE}}$, i.e., $E_{\mathrm{A}}=0-E_{\mathrm{RE}}$. Thus, potential in this paper refers to $\mathrm{V}$ vs $\mathrm{Ag} / \mathrm{AgCl}$. In period $\mathrm{B}$, all WEs were disconnected from each other to achieve an individual open circuit condition and electrode potential of each WE, $E_{\mathrm{B}}$, was measured. Typical intervals of period A and B were $1 \mathrm{ks}$ and $100 \mathrm{~s}$, respectively. $E_{\mathrm{B}}$ was recorded from $100 \mathrm{~s}$ after changing to period B.

To verify the coupling condition of each WE, polarization curve of iron electrode was measured in the same solution of immersion test with a scanning rate of 1 $\mathrm{mV} \mathrm{s}^{-1}$.

\section{Results and discussion}

Fig. 4a shows the time-transient of the coupling current of an individual WE

placed in an artificial crevice with $d_{\mathrm{Gap}}=1.0 \mathrm{~mm}$ during the immersion test. In the initial $5 \mathrm{ks}$, the coupling current was anodic on WE0 at the bottom of the crevice and WE9 near the opening. This tendency was reproducible in other cases, as shown later. 
However, the coupling current on the other WEs was distributed randomly to anodic or cathodic at this stage. After 5 ks the coupling current on WE1-8 showed a sequential transition, i.e., cathodic current increased with time, reaching a cathodic peak, and then decreased and changed to anodic current sequentially from WE2 to WE8. After 90 ks, all WEs showed an almost steady current. At $240 \mathrm{ks}$, a small anodic current on WEs 0-8 was coupled with a large cathodic current on WE9.

Transition of WEs to the anode or cathode is influenced by the condition of local electrolyte solution on each WE. At the initial stage of immersion, anodic dissolution of iron and cathodic reduction of dissolved oxygen (DO) occur simultaneously on each WE because the initial conditions on them are almost identical. Oxygen reduction causes decrease of DO and increase of $\mathrm{OH}^{-}$ions in the crevice solution (alkalization; $2 \mathrm{H}_{2} \mathrm{O}+\mathrm{O}_{2}+4 \mathrm{e}^{-}=4 \mathrm{OH}^{-}$). On the other hand, $\mathrm{OH}^{-}$ions are consumed by hydration reaction of iron ions produced by the anodic dissolution reaction $\left(\mathrm{Fe}^{2+}+2 \mathrm{OH}^{-}=\mathrm{Fe}(\mathrm{OH})_{2}\right)$. Change in $\mathrm{pH}$ in the crevice is thus competitive. Alkalization proceeded smoothly at the bottom of the crevice compared with that near the opening because $\mathrm{OH}^{-}$ions accumulate inside the crevice. For this reason, WEs in 
the crevice tended to act as a cathode and WE9 near the opening tend to act as an anode until DO inside the crevice was consumed. At the initial stage, a small difference in the local environment on each WE can cause anodic or cathodic contribution. For example, slightly rapid alkalization at the bottom of the crevice may suppress reduction reaction of DO on WE0 and thus the reaction rate of the oxygen reduction on the next WE1 becomes dominant because the residual DO was supplied from the bottom of the crevice. Reduction reaction also causes decrease in DO in the crevice from the bottom to the opening and thus cathodic contribution of WE(n) can not be maintained in turn. When a considerable amount of DO in the crevice was consumed, WEs in the crevice except for WE9 changed to anode after ca. 100 ks, as shown in Fig. 4a.

Transition of immersion potential of all WEs in period A (explained in Fig. 3), $E_{\mathrm{A}}$, is shown in Fig. 4b. $E_{\mathrm{A}}$ dropped to ca. $-0.65 \mathrm{~V}_{\mathrm{Ag} / \mathrm{AgCl}}$ at the initial stage, gradually increased until ca $90 \mathrm{ks}$, and then remained around $-0.55 \mathrm{~V}_{\mathrm{Ag} / \mathrm{AgCl}}$. Although the RE was placed outside the crevice, $E_{\mathrm{A}}$ reflected the average potential of WEs in the crevice because of high electric conductivity of the test solution. The initial cathodic shift of $E_{\mathrm{A}}$ around $-0.65 \mathrm{~V}_{\mathrm{Ag} / \mathrm{AgCl}}$ thus reflects the dominant ratio of cathodic area on all WEs, and 
successive anodic shift to $-0.55 \mathrm{~V}_{\mathrm{Ag} / \mathrm{AgCl}}$ reflects the anodic corrosion condition on WEs 0-8. This was confirmed from the polarization curve of iron shown in Fig. 5 in which corrosion potential was ca. $-0.6 \mathrm{~V}_{\mathrm{Ag} / \mathrm{AgCl}}$, and thus the initial value of $E_{\mathrm{A}}$ at -0.65 $\mathrm{V}_{\mathrm{Ag} / \mathrm{AgCl}}$ indicates a cathodically polarized condition and that at $-0.55 \mathrm{~V}_{\mathrm{Ag} / \mathrm{AgCl}}$ indicates an anodically polarized condition. Fig. 4c shows transition of the open circuit potential of an individual WE measured in period $\mathrm{B}, E_{\mathrm{B}}$, under the non-coupling condition. $E_{\mathrm{B}}$ was recorded from $100 \mathrm{~s}$ after WEs were released their coupling condition, i.e., anodic or cathodic polarization condition for each WE. $E_{\mathrm{B}}$ therefore reflects the degree of relaxation of such a polarization condition. In the figure, each WE showed some potential difference in $E_{\mathrm{B}}$ in the noble or less-noble direction from the immersion potential of $E_{\mathrm{A}}$. To emphasize this potential difference, $\Delta E_{\mathrm{B}}=E_{\mathrm{B}}-E_{\mathrm{B}}(\mathrm{avr})$ was plotted as shown in Fig. $\mathbf{4 d}$, where $E_{\mathrm{B}}(\mathrm{avr})$ is the average value of $E_{\mathrm{B}}$ of all WEs. $\Delta E_{\mathrm{B}}$ was in the order of a several tens $\mathrm{mV}$ and showed a sequential transition similar to the current transition shown in Fig 4a. The correlation between coupling current $(I)$ and $E_{\mathrm{B}}$ confirms the polarization states of each WE under the coupling condition. 
Fig. 6 shows transition of specimen surfaces of WEs during the immersion test. A dark and continuous trace of corrosion product was formed on the bottom half of each specimen from WE2 to WE9 until 50 ks. After 50 ks, corrosion product slightly increased. This trace seems to be formed at the boundary of the upper half and lower half of the surfaces of WEs and is related to the internal flow of the gap solution, i.e., upper inflow of the test solution and lower outflow to the outside of the gap. This flow not only causes deposition of corrosion product between both flows but also supplies DO into the crevice, as discussed later.

To analyze spatial $\mathrm{pH}$ distribution in the crevice, an agar strip containing BTB was embedded in the aclylic plate covering the artificial crevice. The results of the BTB test are shown in Fig. 7. The initial color of BTB was yellow because the test solution was weakly acidic due to dissolution of carbon dioxide. After $10 \mathrm{ks}$, the color of BTB gradually changed to blue from the bottom toward the opening of the crevice. After 100 ks, most of the agar in the crevice changed to blue. The expansion of the blue part of BTB with immersion time corresponds to the progression of alkalization due to reduction of DO in the gap solution. It was also confirmed that the transition time of 
color change from yellow to blue corresponded to the transition time of coupling current from cathodic to anodic current on each WE shown in Fig. 4a. The actual pH range of the gap solution in the crevice was, however, not specified because the color of BTB changes to blue at $\mathrm{pH}$ above 7.5.

Coupling current also depended on the dimension of the crevice and direction of the WE surface and gap opening. For example, Fig. 8 shows a comparison of current transitions for three gaps, $d_{\text {Gap }}=0.8,1.0$, and $1.5 \mathrm{~mm}$. In all experiments, sequential transition from cathodic to anodic current on WEs2-8 from the bottom to opening of the crevice was observed. Cathodic current observed for $d_{\mathrm{Gap}}=0.8 \mathrm{~mm}$ was smaller than the others. The duration to reach a cathodic peak current on each $\mathrm{WE}(n), t_{\mathrm{p}}(n)$, became short with increase in $d_{\text {Gap. }}$. For example, $t_{\mathrm{p}}(8)$ was 110,60 and $45 \mathrm{ks}$ for $d_{\mathrm{Gap}}=0.8,1.0$ and 1.5 $\mathrm{mm}$, respectively. In the crevice with a gap of less than $0.5 \mathrm{~mm}$, sequential transition of the coupling current was not clearly observed. On the other hand, sequential transition was also not observed for a gap wider than $2.0 \mathrm{~mm}$ because the condition inside the gap was closed to the bulk condition. Slower transition of coupling current in the narrower gap shown in Fig. 8a was thus influenced by not only smaller ionic coupling current but 
also slower mass transport in the narrower gap. Generally crevice corrosion has been studied for the case of a gap in the order of several tens of $\mu \mathrm{m}$ [20], and thus the coupling current transition found in this study may not be observed for these cases. Typical current transients of WEs 1-8 and WE9 are schematically shown in Fig. 9. For quantitative analysis, characteristic parameters of cathodic current charge for each $\mathrm{WE}(n), Q_{\mathrm{C}}(n)$, and the time at the cathodic current peak, $t_{\mathrm{p}}$, are defined as shown in the figure. The values obtained for the data shown in Fig. $\mathbf{9}$ are summarized in Table $\mathbf{1}$ with a distance between the center of each WE and the bottom of the crevice, $x$. $Q_{\mathrm{C}}(9)$ could not, however, be obtained because the time when the sequential transition was completed could not be determined. $Q_{\mathrm{CT}}$ was summation of $Q_{\mathrm{C}}(0-8), Q_{\mathrm{DO}}$ is a charge which is necessary to reduce all dissolved oxygen in the gap solution without incoming and outgoing calculated from the volume of artificial crevice and the saturated DO value in $3 \mathrm{wt} \% \mathrm{NaCl}$ solution at $25^{\circ} \mathrm{C}$.

The plot of $t_{\mathrm{p}}$ against $x$ shown in Fig. 10 reveals an almost linear relationship between them. The time of current peak acquired is shorter in the case of wider gap. 
This is related to the progression of alkalization in the gap due to reduction of DO, as discussed later.

In the plot of $Q_{\mathrm{C}}(n)$ against $n$ shown in Fig. 11, three regions can be distinguished as follows. $Q_{\mathrm{C}}(0-1$ or 2$)$ was small because these WEs behaved as anode rather than cathode during the sequential current transition. $Q_{\mathrm{C}}(2$ or 3-6) was larger than the former and tend to increase with $n$. $Q_{\mathrm{C}}(7-8)$ was larger than others due to a larger contribution of the cathodic current for DO reduction supplied from the opening of the gap. The contribution of DO near the opening was larger for a wider gap, indicating efficient DO supply through a wider opening. On the other hand, values of $Q_{\mathrm{C}}(0-6)$ did not depend on $d_{\text {Gap }}$. Since the ratio of total cathodic charge $Q_{\mathrm{CT}}$ to $Q_{\mathrm{DO}}$, is as large as 6.4 to 11.5 as shown in Table 1, most of DO reduced in the gap was introduced from the outside of the crevice and caused considerable alkalization in the crevice.

From these results, sequential transition of coupling current can be interpreted as shown in Fig. 12 in which spatial distribution of $\mathrm{pH}$, DO concentration and anodic or cathodic current contribution are schematically drawn as a function of distance from the bottom of the crevice, $x$. At the initial stage of immersion, both anodic dissolution of 
iron and cathodic reduction of DO proceed on all WEs. The ratio of anodic and cathodic contributions on each WE seems to be random. Reduction reaction of DO causes alkalization of the gap solution from the bottom of the crevice, probably because of slow mass transportation at the bottom of the crevice. Once alkalization becomes dominant at the bottom of the crevice, iron dissolution reaction is suppressed and thus DO reduction becomes dominant. This triggers sequential current-transition in the gap within 10 ks. As shown in Fig. 12, inflow of the test solution from the outside of the gap supplies DO. Cathodic reaction of DO causes accumulation of alkaline solution from the bottom of the gap, resulting in a $\mathrm{pH}$ gradient in the gap which moves from the bottom to the opening of the gap. Suppression of iron dissolution accompanying this movement makes DO reduction reaction predominant on each WE(n) surface. However, the progression of alkalization also suppresses DO reduction reaction due to increase in $\mathrm{OH}^{-}$concentration. This causes the appearance of a cathodic current peak. The cathodic current peak moves from the bottom to the opening with time, as shown with the arrow in the figure. After the current has peaked, both the anodic dissolution current and DO reduction current become low. After completion of the current transient, a small anodic 
coupling current was observed on WEs (WE(n-1) in Fig.12) in the gap to match the large cathodic current observed on WE9 due to sufficient supply of DO and weakened alkalization effect. In the above explanation, however, there is the exception of WE0-2, which always showed anodic contribution. Supply of DO to these WEs probably was not sufficient and they had to become anode to match with the other cathode.

From these discussion, it is supposed that DO concentration in the gap is not low and that alkalization of the gap solution proceeds rather than acidification in the present experimental conditions. These phenomena are observed for a crevice with an intermediate gap and differ from that proposed for the case of narrower gap such as micro meter order. As seen in Table 1, a considerable amount of DO could be absorbed into such a crevice with an intermediate gap. In some cases, absorption of DO into the gap causes moderation of the corrosion rate in the gap, especially around the opening, and enables cathodic contribution of this area coupled with anodic dissolution occurring on other areas.

\section{Conclusion}


The multichannel electrode method was applied to crevice corrosion of iron

immersed in sodium chloride solution. Ten iron electrodes were placed in an artificial crevice with one side exposed to the solution with a constant gap distance. Using this condition, spatial distribution and time transient of the coupling current between ten electrodes were measured, and the following conclusions were obtained.

1) WE0 located at the innermost crevice showed an anode coupling current at all times during immersion. WE1-8 located at the middle of the crevice initially showed a cathode coupling current, which sequentially changed to a anodic current. On each WE, the coupling current increased cathodically, reached a cathodic peak, decreased, and then changed to an anodic current. WE9 near the opening showed different behavior, i.e., WE9 initially showed an anodic coupling current to match the cathodic coupling current of WE1-8 and later showed a considerable cathodic current of DO reduction supplied from the opening of the crevice to match the anodic coupling current of other WEs. 
2) The progression of alkalization caused by DO reduction and dissolution of iron in the crevice were observed by using a pH indicator and iron ion indicator reagents. From quantitative analysis of the cathodic charge consumed for DO reduction reaction in the gap, it was confirmed that considerable DO was taken into the gap from outside the gap due to inflow and outflow of solution.

3) Alkalization of the gap solution from the bottom to the opening of the gap proceeded at an almost constant rate probably due to steady inflow of solution into the gap. The rate of alkalization decreased with decrease in gap thickness in the range of 0.8 to 1.5 $\mathrm{mm}$, indicating a slow internal flow rate in the narrow gap. Beyond this range of gap thickness, sequential transition of the coupling current was not clearly observed.

\section{References}

[1] A. J. Betts, L. H. Boulton, Br. Corros. J., 28 (1993) 279.

[2] B. F. Brown, C. T. Fujii and E. P. Dahlberg, J. Electrochem. Soc., 116 (1968) 218. 
[3] M. Pourbaix, in: J. C. Scully (Ed.), The Theory of Stress Corrosion Cracking in Alloys, Proc., NATO Ericeira Conference, Brussels, Belgium, 1971, p. 17.

[4] H. W. Pickering, R. P. Frankenthal, J. Electrochem. Soc., 119 (1972) 1297.

[5] J. W. Oldfield, W. H. Sutton, Br. Corros. J., 13 (1978) 13.

[6] S. M. Sharland, Corros. Sci., 27 (1987) 289.

[7] G. F. Kennell, R. W. Evitts, K. L. Heppner, Corr. Sci., 50 (2008) 1716.

[8] F. M. Song, N. Sridhar, Corr. Sci., 50 (2008) 70.

[9] S. Nagarajan, N. Rajendran, Corr. Sci., 51 (2009) 217.

[10] M. I. Abdulsalam, Corr. Sci., 47 (2005) 1336.

[11] J. R. Galvele, Corr. Sci., 47 (2005) 3053.

[12] H. W. Pickering, Corros. Sci., 29 (1989) 325.

[13] K. Cho, M.I. Abdulsalam, H.W. Pickering, J. Electrochem. Soc., 145 (1998) 1862.

[14] K. Fushimi, A. Naganuma, K. Azumi, Y. Kawahara, Corros. Sci., 50 (2008) 903.

[15] Y. J. Tan, Prog. Org. Coat., 19 (1991) 89. 
[16] Y. J. Tan, S. T. Yu, Prog. Org. Coat., 19 (1991) 257.

[17] Y. Tan, Corrosion, 54 (1998) 403.

[18] Y. J. Tan, S. Bailey, B. Kinsella, Corros. Sci., 43 (2001) 1905.

[19] Y. J. Tan, S. Bailey, B. Kinsella, Corros. Sci., 43 (2001) 1919.

[20] R. Kain, National Association of Corrosion Engineers, 40 (1984) 313.

\section{Figure Captions}

Table 1 Cathodic charge $\left(Q_{\mathrm{C}}\right)$ and current peak time $\left(t_{\mathrm{P}}\right)$ of an individual WE $(n)$ at a distance $x$ from the crevice bottom as a function of crevice gap $\left(d_{\mathrm{Gap}}\right)$. $Q_{\mathrm{CT}}$ is summation of cathodic current transient on WE1 - WE8, DO is the amount of dissolved oxygen in the gap at the saturated concentration, and $Q_{\mathrm{DO}}$ is the charge necessary to reduce DO.

Fig. 1 Preparation of a sample composed of ten iron electrodes: (a) side view, (b) front view. The closing and the opening of the gap correspond to sample 0 and sample 9 , respectively. 
Fig. 2 Brief electronic circuit of the multichannel electrode system.

Fig. 3 Sequence of relay switching in periods A and B. In period A, WEs are connected to a ground level and one of the WEs is sequentially connected to virtual ground to measure the coupling current. In period B, all WEs are disconnected to each other from measure open circuit potential of an individual WE.

Fig. 4 Transition of (a) coupling current, $I$, (b) immersion potential of all WEs during period $\mathrm{A}, E_{\mathrm{A}}$, (c) open circuit potential of an individual $\mathrm{WE}$ during period $\mathrm{B}, E_{\mathrm{B}}$, and (d) potential difference of each WE against average potential of all WEs, $\Delta E$, measured for ten WEs with gap thickness of $1.0 \mathrm{~mm}$ immersed in $5.1 \times 10^{2} \mathrm{~mol} \mathrm{~m}^{-3} \mathrm{NaCl}$ solution. At Fig. 4a, broken line indicates base line.

Fig. 5 Polarization curve of iron electrode in $5.1 \times 10^{2} \mathrm{~mol} \mathrm{~m}^{-3} \mathrm{NaCl}$ solution. Sweep rate was $1 \mathrm{mV} \mathrm{s}^{-1}$. 
Fig. 6 Photos of WEs at various immersion times during the experiment for which results are shown in Fig. 4.

Fig. 7 Photos of WEs placed in an artificial crevice with a $1.0 \mathrm{~mm}$ gap immersed in $5.1 \times 10^{2} \mathrm{~mol} \mathrm{~m}^{-3} \mathrm{NaCl}$ solution. Agar containing BTB was embedded in the acrylic plate for forming a crevice to indicate $\mathrm{pH}$ change in the gap solution by blue color.

Fig. 8 Transition of coupling current, $I$, measured for iron electrodes placed in artificial crevices. (a) $d_{\text {Gap }}=0.8 \mathrm{~mm}$, (b) $1.0 \mathrm{~mm}$ and (c) $1.5 \mathrm{~mm}$.

Fig. 9 Typical current transition on WE1-8 and WE9.

Fig. 10 Dependence of peak time $\left(t_{\mathrm{P}}\right)$ on distance $(x)$ of each WE from the bottom of the gap and crevice gap $\left(d_{\mathrm{Gap}}\right)$. 
Fig. 11 Cathodic charge $\left(Q_{C}\right)$ of individual WEs as a function of location of WE $(n)$ in the artificial crevice.

Fig. 12 (a) explanation of sequential current transition on WE2-8, (b) formation of cathodic current wave in the gap. 
a)

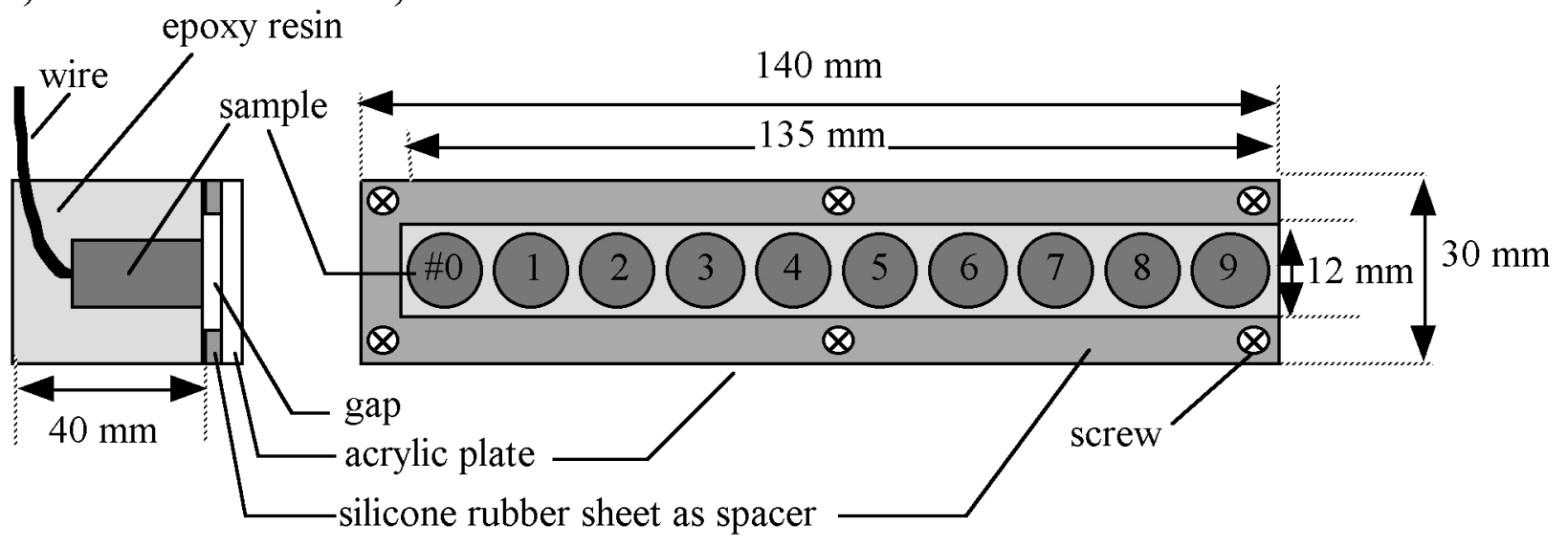




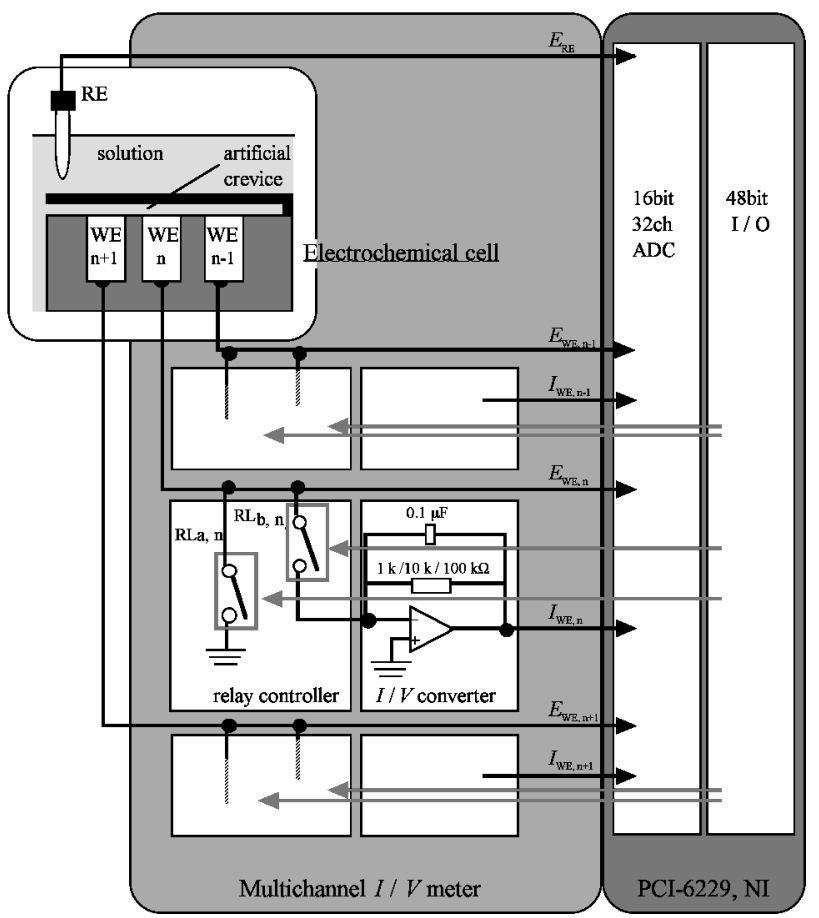



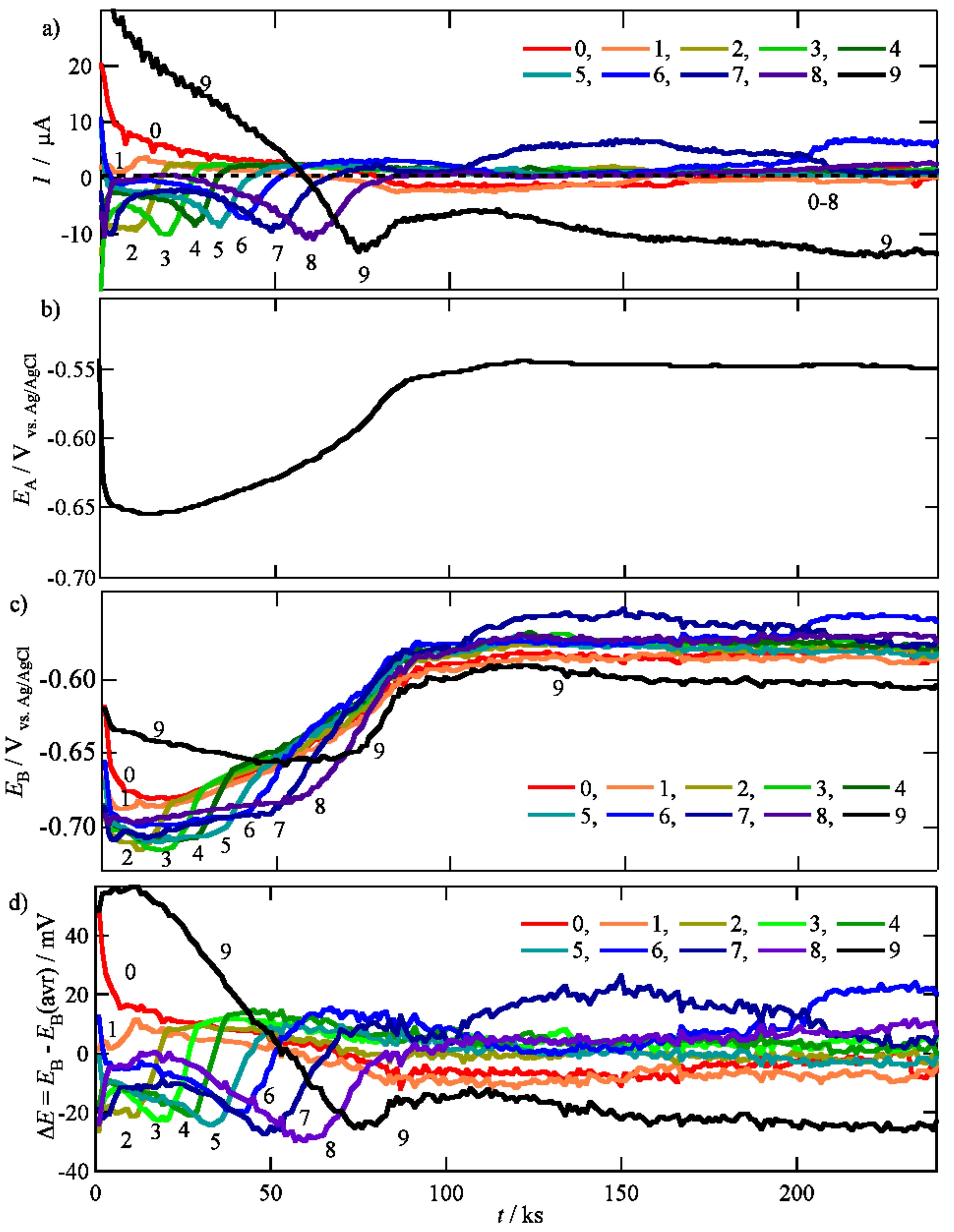


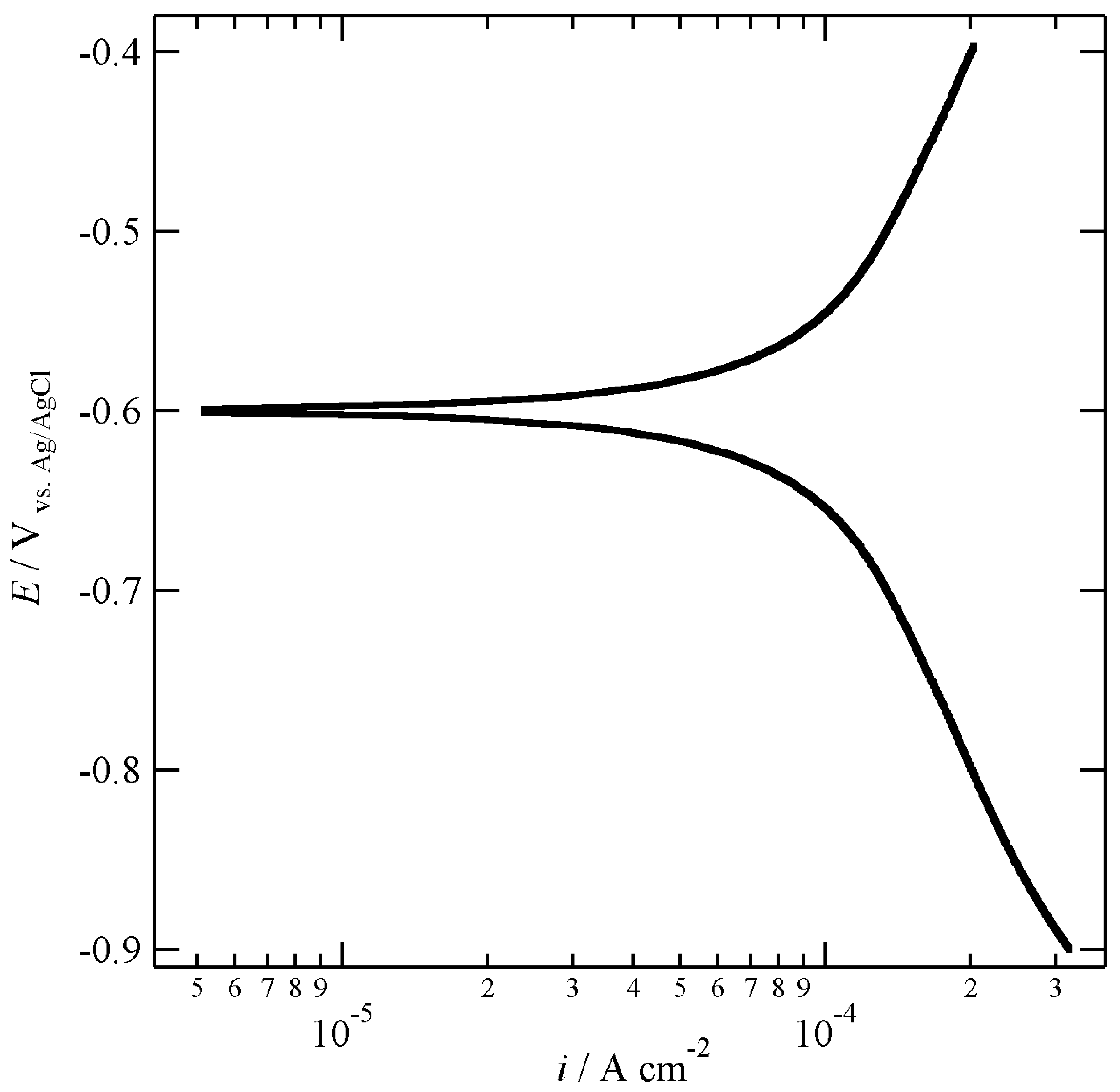


$0 \mathrm{ks}$

$50 \mathrm{ks}$

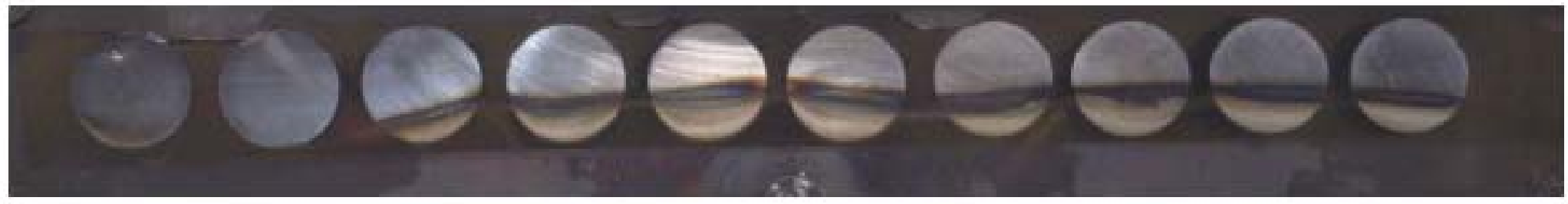

$150 \mathrm{ks}$

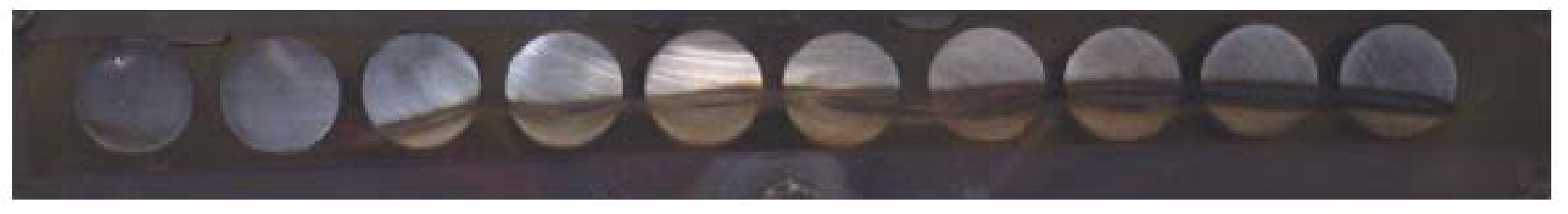

$240 \mathrm{ks}$

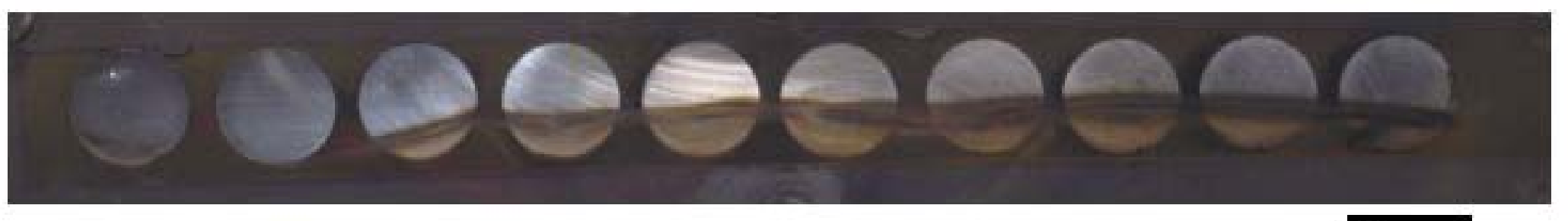

$10 \mathrm{~mm}$
$\begin{array}{ll}0 & 1\end{array}$
2
3
4
5
6
7
8
9 
$0 \mathrm{ks}$

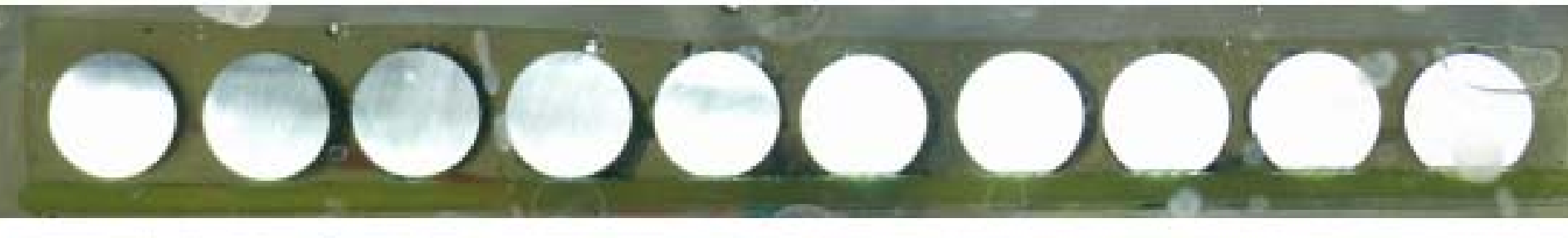

$10 \mathrm{ks}$

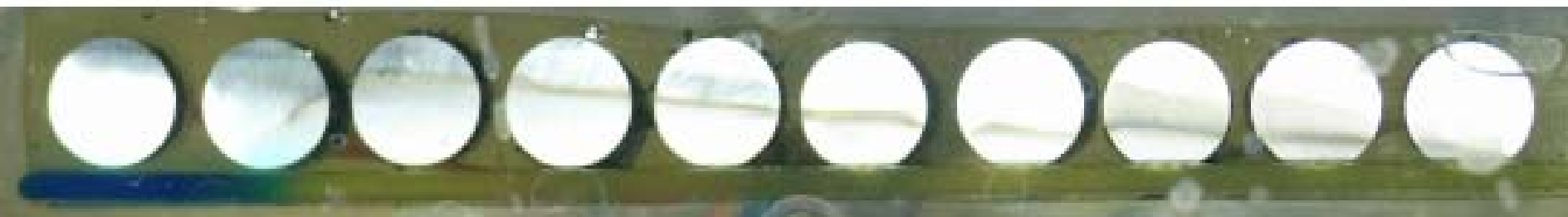

$20 \mathrm{ks}$

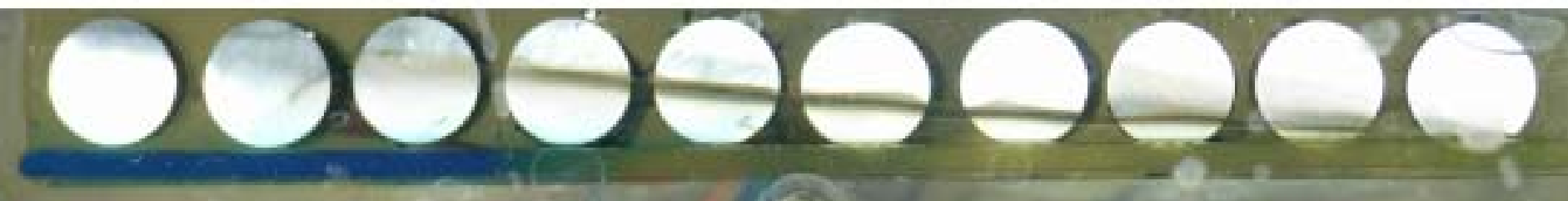

a)

$50 \mathrm{ks}$

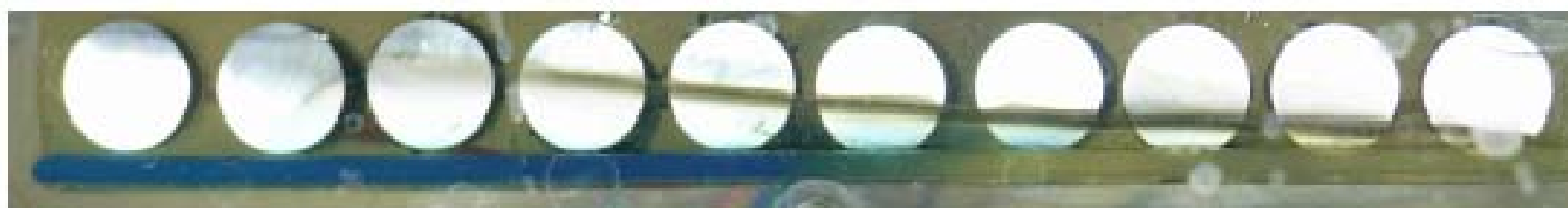

ail

$100 \mathrm{ks}$
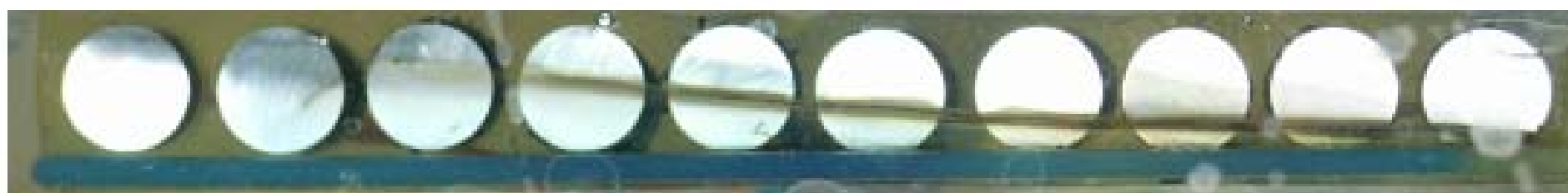

(a)
0
2
3
4
5
6
7
8
9 


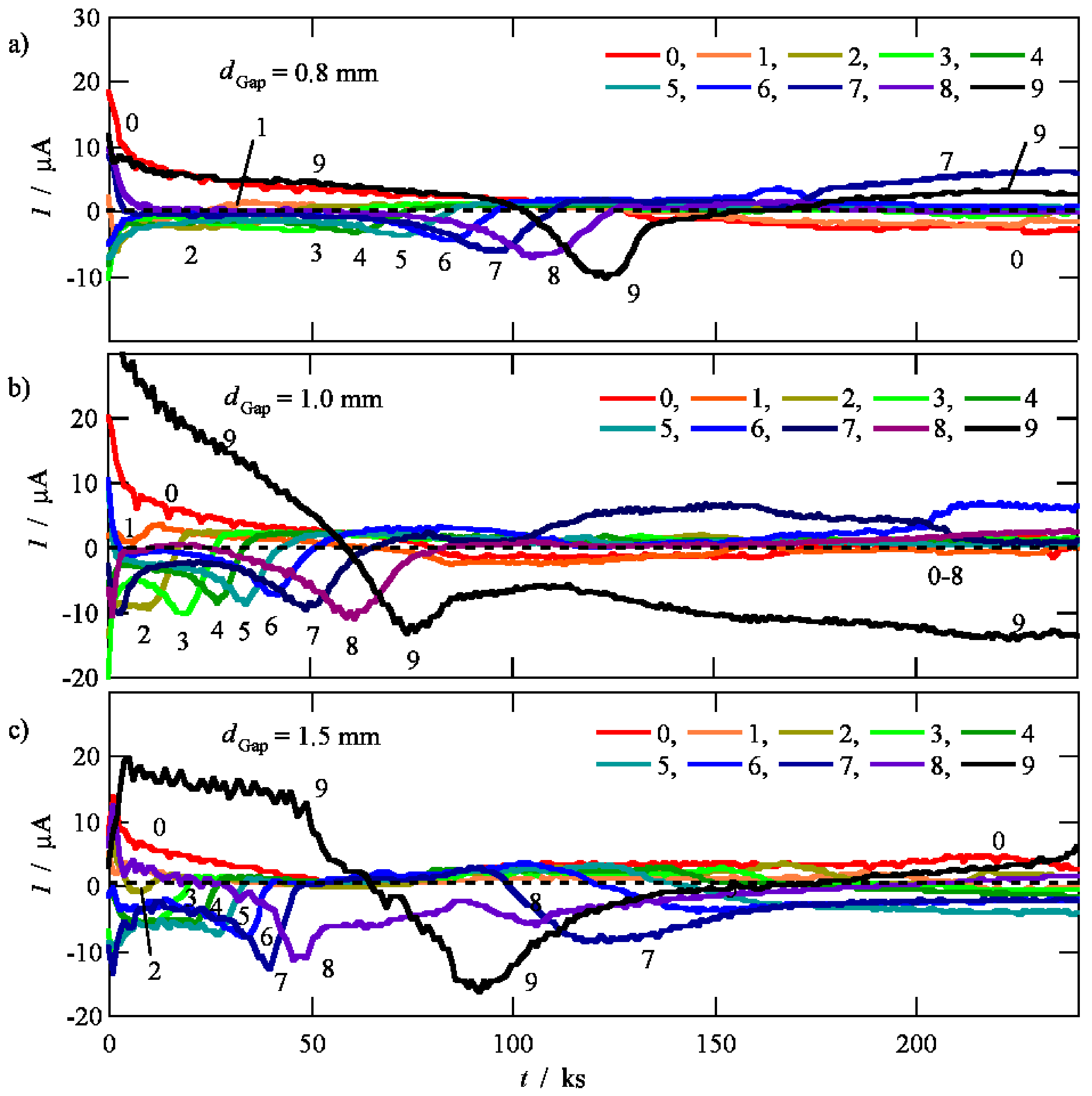




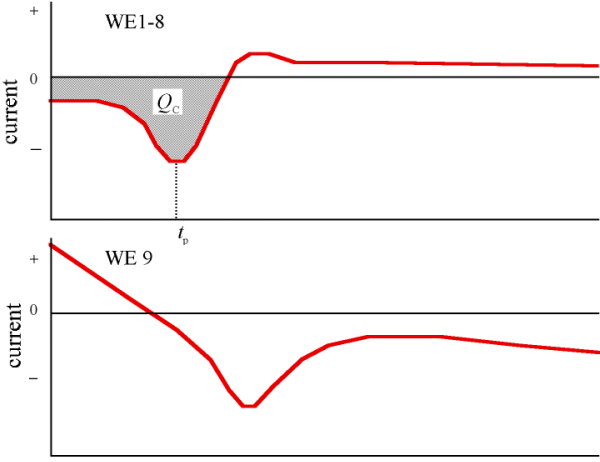

time 


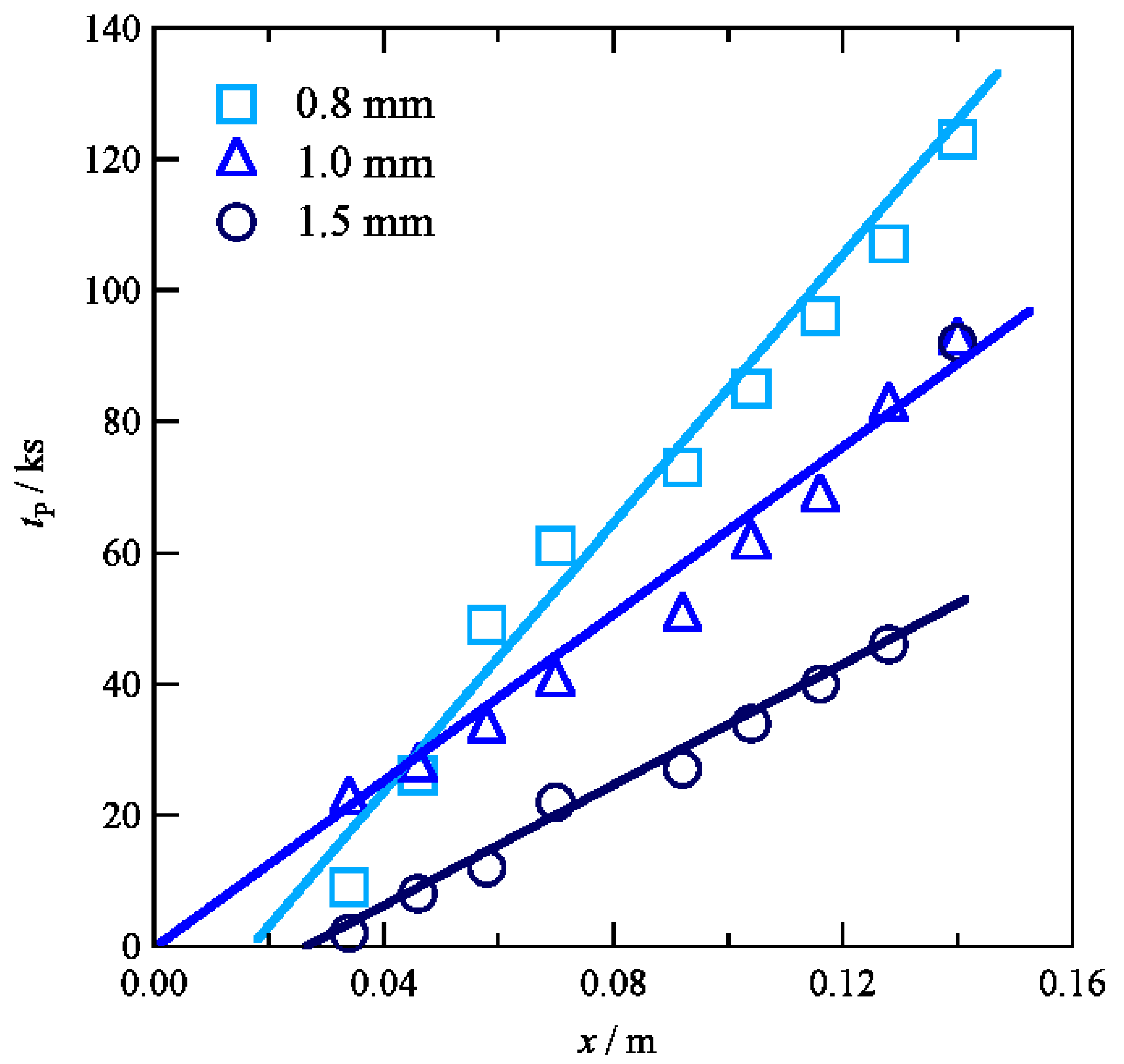




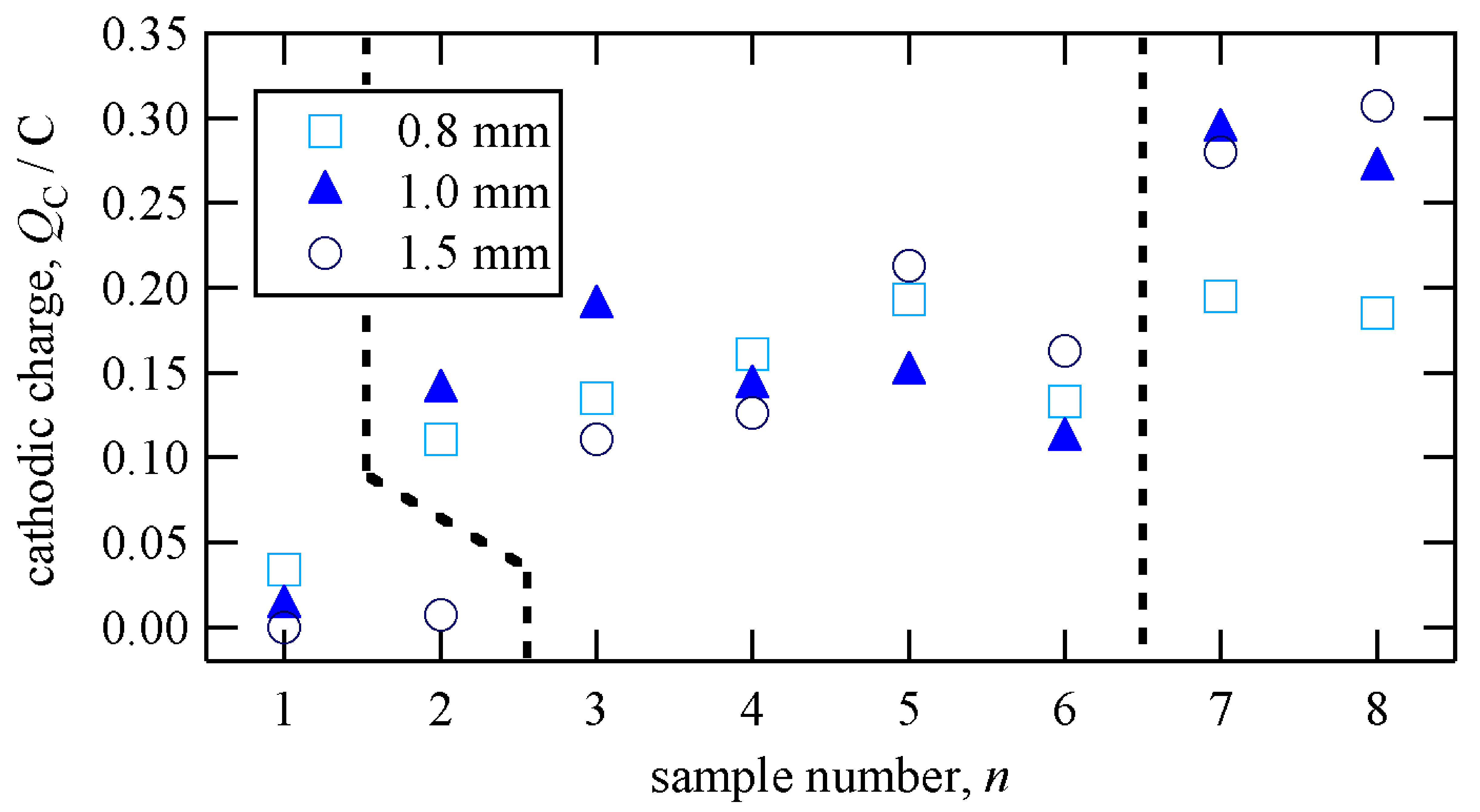


